\title{
EXPLICITANDO LA INTERRELACIÓN ENTRE LAS ACTIVIDADES DE APRENDIZAJE, EL PROCESO DE EVALUACIÓN Y LA ADQUISICIÓN DE COMPETENCIAS
}

\author{
(EXPLICATING THE INTER-RELATIONSHIP BETWEEN LEARNING ACTIVIES, THE \\ EVALUATION PROCESS, AND THE ACQUISITION OF COMPETENCES)
}

\section{Ana-Elena Guerrero-Roldán}

M. Antonia Huertas

Enric Mor

M. Elena Rodríguez

Universitat Oberta de Catalunya (España)

\section{RESUMEN}

El sistema universitario europeo ha definido los grados en base a un conjunto de competencias generales y específicas que deben ser adquiridas por los estudiantes. Las competencias establecidas deben reflejarse en las asignaturas de grado, siendo necesario alinear cada competencia con las actividades que realiza el estudiante. A su vez, los criterios de evaluación de cada actividad deben reflejar el grado de adquisición de cada competencia. Tanto la calificación emitida como el feedback que recibe el estudiante deben estar relacionados con las competencias para poder mostrar al estudiante su progresión. En este artículo se presenta el proceso de trabajo para alinear las competencias de grado con las de asignatura, sus actividades, criterios de evaluación y resultados de aprendizaje. La principal aportación es la interrelación realizada, concretando y clarificando las competencias específicas, tanto para los docentes como los estudiantes en el caso de la asignatura de Lógica.

Palabras clave: educación superior, competencias, proceso de enseñanza y aprendizaje, actividades, proceso de evaluación, feedback.

\begin{abstract}
The European university system defines degrees according to a series of general and specific competences that should be acquired by students. These established competences should be reflected in the degree subjects - as it is necessary to align each competency with activities performed by students. At the same time, the evaluation criteria of each activity should reflect the level of acquisition of each competence. Both the qualification that is granted as well as the feedback that the student receives should be related to the competences, i.e. to
\end{abstract}


show the student's progression. In this article, we present a working procedure to align the degree competences with the course competences, its activities, the evaluation criteria, and the learning outcomes. The main contribution of this paper lies in its analysis of the relationship established between the various elements, namely those which specify and clarify the specific competences required for both teachers and students in a degree course on Logic.

Keywords: higher education; competences; teaching-learning process; activities; assessment process; feedback.

La introducción progresiva de las Tecnologías de la Información y la Comunicación en contextos educativos se ha convertido en una pieza clave para la construcción y modelación de los procesos de enseñanza y aprendizaje, tanto en modelos presenciales, mixtos como a distancia. Actualmente los docentes pueden disponer de una gran variedad de herramientas y sistemas que permiten fomentar, entre otras, la comunicación, la colaboración en red y la gestión de los recursos de aprendizaje (Fainholc, 2008). La introducción de estas herramientas proporciona al docente la posibilidad de trabajar y evaluar a los estudiantes de forma muy variada, mejorando en la mayoría de casos, la calidad de las asignaturas impartidas, su metodología, las actividades y la manera de evaluar las mismas. Cada vez más, las instituciones educativas disponen de herramientas para favorecer un feedback cualitativo del trabajo que realiza el estudiante, aunque todavía se mantiene la calificación numérica.

En el marco universitario previo al Espacio Europeo de Educación Superior (EEES), el proceso de evaluación se centraba en el aprendizaje de contenidos con una calificación numérica como un medio para determinar el grado de consecución de los objetivos de cada estudiante. A raíz del EEES, el sistema universitario pasa a centrarse en un modelo educativo que parte del concepto de actividad y sitúa en el centro del proceso de aprendizaje al propio estudiante. Parcialmente este modelo deja de lado el logro de objetivos, para centrarse en uno donde priman las competencias a alcanzar y el grado de adquisición de cada una (ya sea general o específica). En este marco, los estudiantes son evaluados por competencias (procesos) y no sólo por contenidos (conocimientos). El estudiante debe adquirir progresivamente un conjunto de competencias que lo harán un profesional competente poniendo en práctica un conjunto de habilidades y destrezas que muestran su aprendizaje. Este cambio de paradigma, supone un replanteamiento del proceso de evaluación para alinearlo con el logro de competencias (Avargil et al., 2012).

El proceso que se describe en este artículo nace de la necesidad de revisar y analizar, por parte de un conjunto de docentes, el trabajo basado en competencias que se ha realizado en estos últimos años de implantación del grado. Si bien los 
grados tienden a seguir lo definido en las memorias, se hace necesaria una reflexión a nivel de asignatura, con visión top-down y a su vez botton up, que implica un análisis y revisión de las competencias específicas que se están trabajando, y cómo éstas se están desarrollando y evaluando a nivel de asignatura.

\section{MARCO TEÓRICO}

El sistema universitario está implantando progresivamente los grados siguiendo las directrices del EEES y tipificando las competencias más apropiadas para cada titulación. En consecuencia está desplegando asignaturas que centradas en la adquisición de competencias mediante el trabajo y la realización de diferentes actividades, prácticas y ejercicios, tienen como objetivo principal dotar a los estudiantes de unas competencias generales y de competencias más específicas en función de la titulación y la tipología de asignaturas que imparten.

Siguiendo este trabajo, se hace necesario el replanteamiento de cómo se realiza el proceso de evaluación (tanto parcial como globalmente). Esta necesidad conlleva analizar por parte de los docentes, las competencias que se trabajan, en qué actividades, cómo se realiza la evaluación, cuando se evalúa, de qué instrumentos se dispone y qué feedback se proporciona al estudiante sobre su proceso de aprendizaje en relación a las competencias y habilidades establecidas. También implica trabajar el retorno personalizado que se proporciona a cada estudiante en cada una de las actividades que realiza desde el punto de vista cualitativo. Se mantiene la calificación numérica como un mecanismo establecido pero se añade el concepto de nivel o grado competencial como un medio gradual del logro del trabajo (Scriven, 1974).

Una competencia se define como una combinación de atributos (respecto a conocimientos, aptitudes y destrezas) que describen el nivel o grado de suficiencia que una persona es capaz de desarrollar y poner en práctica una actividad. Este término se asocia a otros conceptos con significados muy similares como el de capacidad, atributo, destreza o habilidad y comporta de forma inherente la puesta en práctica del conocimiento (González et al., 2003). En cada grado, las competencias pueden ser clasificadas como generales y específicas estableciéndose además el nivel deseado de adquisición de cada una de ellas (básico, medio y avanzado).

Parafavorecer la adquisición de competencias por parte del estudiante es necesario replantear las actividades que deben realizar así como su proceso de evaluación. Éstas son ahora el punto de referencia para fomentar el trabajo de las habilidades y destrezas de cada estudiante. Mediante las actividades y las prácticas los estudiantes deben mostrar las competencias adquiridas. El proceso de evaluación de las mismas 
debe ser minucioso y sistemático, de tal forma que permita mejorar el aprendizaje del estudiante a través de cada actividad. Como se indica en JISC (2007) "el proceso de evaluación es fundamental para la enseñanza y el aprendizaje. Lo evaluado define que se ha enseñado y cómo se ha aprendido. El proceso de evaluación da forma a la práctica institucional y afecta a la participación del estudiante en su proceso de aprendizaje”. La evaluación puede ser categorizada en diagnóstica, formativa y sumativa dependiendo del momento del curso en que se lleva a cabo (Díaz, 2002). En este sentido, el modelo de evaluación que más se ajusta a un proceso de aprendizaje basado en competencias es un modelo de evaluación formativa (López-Pastor, 2010). La evaluación formativa se lleva a cabo durante el proceso de aprendizaje permitiendo al estudiante mejorar sus actividades y su nivel de comprensión (Iram et al, 2011; Scriven, 1967). Una mayor implicación del estudiante puede suponer una mejora en su proceso de aprendizaje (Mostrom, 2012). Una creciente evidencia indica que una evaluación formativa bien diseñada y aplicada promueve un aprendizaje mas efectivo (Nicol, 2006; Sharpe et al., 2006). Mediante la evaluación formativa el proceso de adquisición de competencias es completamente dinámico e iterativo para el estudiante. Al finalizar cada actividad el estudiante obtiene una calificación y, a su vez, un feedback cualitativo y personalizado que le permite analizar los puntos débiles y fuertes de su trabajo para mejorar su proceso de aprendizaje. El feedback recibido indica al estudiante las unidades que puede repasar y las actividades de autoaprendizaje que puede realizar para reforzar nuevamente los contenidos trabajados mediante las actividades y también las competencias trabajadas. Como se indica en Burgos (2006), la evaluación y el feedback son los mecanismos más utilizados y más eficientes para la adaptación puesto que permiten la reconducción del proceso de formación. Chen et al. (1999) comentan que el feedback mejora el aprendizaje y reduce el sentimiento de aislamiento. Halttunen et al. (2000) indican que el feedback aumenta la motivación del aprendizaje e invita a la exploración y la experimentación de nuevas actividades. El feedback permite acomodar diferentes concepciones del aprendizaje (Lytras et al., 2005) y además puede favorecer el proceso de aprendizaje basado en competencias.

Actualmente la evaluación de competencias es realizada por gran parte de docentes a través de la rúbrica (Moskal, 2000). Una rúbrica se define cómo "una herramienta de evaluación que identifica ciertos criterios para un trabajo, incluyendo gradaciones de escala calidad para cada trabajo" (Goodrich, 2000). La rúbrica de una competencia permite proporcionar unos criterios de evaluación en base a unos niveles establecidos y/o en base a tramos de calificaciones. En la actualidad están siendo ampliamente aplicadas por el profesorado en diferentes asignaturas (Torres et al., 2010; Navarro et al., 2011; Marín et al., 2012). Su uso está muy extendido entre 
la comunidad docente aunque dichas experiencias se centran en tipificar los criterios de evaluación para establecer el nivel de consecución de los objetivos (Tierney et al., 2004). Las rúbricas incluyen gradaciones de calidad y, en la mayoría de casos, la interrelación entre las competencias de grado, las propias de la asignatura y su visibilidad en las actividades es escasa. La vinculación e interrelación de todos los elementos en el proceso es esencial para asegurar la coherencia y un buen proceso de enseñanza y aprendizaje centrado en la adquisición de competencias. En particular, se deben considerar tanto las competencias como sus actividades y sus indicadores de puesta en práctica para evaluarlas (Richey et al., 2011).

\section{CONTEXTO}

El presente trabajo se ha llevado a cabo en la Universitat Oberta de Catalunya ${ }^{1}$ que se caracteriza por ser una universidad a distancia y totalmente virtual con un modelo educativo centrado en la actividad que realiza el estudiante. El trabajo realizado se centra concretamente en el grado de Ingeniería Informática y en la asignatura de Lógica. Ésta es una asignatura de formación básica de 6 créditos ECTS y, por lo tanto, debe ser cursada por todos los estudiantes de dicho grado.

Lógica es una asignatura que requiere que el estudiante adquiera un conjunto de habilidades y destrezas para así poder aplicar técnicas específicas de la asignatura como por ejemplo técnicas de razonamiento y validación formal. Va más allá de adquirir un conocimiento teórico. Por ello, el principal objetivo es que el estudiante sea capaz de utilizar la lógica de primer orden en la validación de razonamientos formales. En el contexto de la enseñanza a distancia en un entorno virtual en esta asignatura, los estudiantes disponen de un sistema de tutor inteligente llamado "Asistente", que es un software para la práctica de ejercicios de la mayor parte de los módulos de la asignatura con autocorrección y feedback inmediato. En esta asignatura actualmente se aplica el modelo de evaluación formativa que se basa en la evaluación continua y un examen final presencial. Durante la asignatura los estudiantes deben realizar un conjunto de actividades de evaluación que se denominan Pruebas de Evaluación Continua (PECs), las cuales fomentan, por un lado, el trabajo y la adquisición de habilidades y destrezas (PECs) y, por otro, aseguran la adquisición de los conocimientos básicos. Las PECs se realizan con el asistente y antes de cada una, los estudiantes pueden realizar ejercicios de autoevaluación también con el asistente. Estas actividades conforman la evaluación formativa de la asignatura.

En este contexto se propone realizar la alineación de competencias del grado de Ingeniería Informática, la asignatura de Lógica y sus actividades. Además, a través de cada una de las actividades se propone reflejar las competencias que se trabajan, 
el nivel esperado de cada competencia y los criterios e indicadores de evaluación que muestran la adquisición de competencias.

\section{IMPACTO DE LAS COMPETENCIAS DEL GRADO EN LA ASIGNATURA}

Las competencias establecidas en el grado de Ingeniería Informática se dividen en competencias transversales, generales y específicas. Este trabajo parte de las competencias generales de grado para realizar un proceso de concreción y revisión de dichas competencias y su especificidad en las asignaturas. Entre las competencias de grado establecidas en la memoria nos centramos en dos de ellas, puesto que son las que están directamente relacionadas con la asignatura de Lógica. Estas competencias son las siguientes:

- Capacidad de utilizar los fundamentos matemáticos, estadísticos y físicos para comprender los sistemas TIC.

- Capacidad de analizar un problema en el nivel de abstracción adecuado a cada situación y aplicar las habilidades y conocimientos adquiridos para abordarlo y resolverlo.

Ambas competencias se deben ver reflejadas de forma directa o indirecta en las competencias específicas propias de la asignatura y en sus actividades. No obstante, las competencias específicas van más allá de las generales y a través de ellas se enfatizan algunas habilidades y destrezas de forma más detallada, que posteriormente se concretarán. Las competencias específicas propias de la asignatura que deberá haber adquirido cualquier estudiante al finalizar el curso son las siguientes:

- Aprender a formalizar expresiones del lenguaje natural usando lógica de enunciados y de predicados.

- Adquirir habilidades de validación de razonamientos en lógica de enunciados y de predicados usando el método de deducción natural.

- Adquirir habilidades de validación de razonamientos en lógica de enunciados y de predicados usando el método de resolución.

- Comprender la semántica de la lógica de enunciados y de predicados y saber aplicarla en la validación o refutación de razonamientos. 
- Adquirir los conocimientos básicos de la lógica booleana y saber aplicarla en los circuitos lógicos.

- Conocer el lenguaje de la teoría de conjuntos básica y saber relacionarla con la lógica.

A partir de estas seis competencias se ha realizado un tercer proceso de concreción que consiste en detallar cada una de las competencias específicas en destrezas y habilidades concretas. Después de analizar cada competencia específica y el propio contenido de la asignatura, en el caso de Lógica se han concretado en las siguientes:

1.1 Saber formalizar expresiones del lenguaje natural usando lógica de enunciados.

1.2 Saber formalizar en lógica de predicados expresiones que involucran cuantificadores.

2.1 Capacidad de construir una demostración de deducción natural para validar un razonamiento en lógica de enunciados.

2.2 Capacidad de construir una demostración correcta de deducción natural para validar un razonamiento en lógica de predicados.

3.1 Saber usar el método de resolución para validar razonamientos en lógica de enunciados.

3.2.Saber usar el método de resolución pera validar razonamientos en lógica de predicados.

4.1 Conocer la aplicación de las tablas de verdad a la validación o refutación de razonamientos de lógica de enunciados.

4.2.Saber dar contraejemplos de un razonamiento que no es válido en lógica de predicados.

5.1 Saber manipular algebraicamente las variables y los operadores booleanos y formar tablas de verdad.

5.2 Entender la aplicación de la lógica booleana a los circuitos digitales. 
6.1 Saber definir correctamente conjuntos y condiciones de pertenencia de un elemento a un conjunto.

6.2 Conocer los símbolos formales de la teoría de conjuntos básica.

A raíz de la jerarquía competencial establecida en la memoria de grado y en la propia asignatura, es necesario relacionar una o más competencias con las actividades que se proponen en la asignatura para interrelacionarlas. El nivel de granularidad de cada competencia va disminuyendo y esto nos permite realizar y establecer ahora su correspondencia con las actividades.

\section{LAS COMPETENCIAS EN LAS ACTIVIDADES}

Con el objetivo de vincular las competencias de Lógica con las diferentes actividades de la asignatura se ha realizado un proceso minucioso de análisis de cada actividad. En este caso se ha asignado una o varias competencias en relación a una o varias actividades. Es decir, se han vinculado e interrelacionado competencias y actividades. En algún caso se ha realizado el proceso inverso puesto que la actividad planteada ya tenía una relación directa con la competencia estipulada sin necesidad de realizar una nueva actividad para dicha competencia. Para cada una de las actividades, además de indicar qué competencia se trabaja, se ha incluido hasta qué nivel se trabaja. Esto implica haber definido previamente el nivel mínimo y máximo de cada competencia que cualquier estudiante deberá adquirir en la asignatura de Lógica.

Como se puede observar en la tabla 1, aunque existen tres niveles de cada competencia en algunos casos sólo se deberá lograr un nivel básico. Esto indica que dicha competencia probablemente será ampliada o consolidada en asignaturas posteriores del mismo grado. También indica que una competencia puede ser trabajada en varias actividades $\mathrm{y}$, por consiguiente, no estará completamente adquirida hasta la finalización y superación del conjunto de actividades que la trabajen. La relación entre las competencias de la asignatura, su concreción y nivel, y las actividades en que se trabajan, quedan reflejadas en la siguiente tabla:

\begin{tabular}{|c|c|c|c|c|c|}
\hline Competencia & Concreción & $\begin{array}{c}\text { Nivel } \\
1\end{array}$ & Nivel & \multicolumn{2}{|c|}{ Nivel 3} \\
\hline \multirow{2}{*}{$\begin{array}{l}\text { Aprender a formalizar expresiones del lenguaje } \\
\text { natural usando la lógica de predicados. }\end{array}$} & 1.1 & PEC 1 & PEC 2 & \multirow[b]{2}{*}{$\mathrm{PEC}_{3}$} & \\
\hline & 1.2 & & & & $\mathrm{PEC}_{4}$ \\
\hline
\end{tabular}




\begin{tabular}{|c|c|c|c|c|}
\hline Competencia & Concreción & $\begin{array}{c}\text { Nivel } \\
1\end{array}$ & Nivel 2 & Nivel 3 \\
\hline \multirow{2}{*}{$\begin{array}{l}\text { 2. Adquirir habilidades de validación de } \\
\text { razonamientos en lógica de enunciados y } \\
\text { predicados usando método deducción natural. }\end{array}$} & 2.1 & $\mathrm{PEC} 1$ & & \\
\hline & 2.2 & & $\mathrm{PEC}_{3}$ & \\
\hline \multirow{2}{*}{$\begin{array}{l}\text { 3. Adquirir habilidades de validación de } \\
\text { razonamientos en lógica de enunciados y de } \\
\text { predicados usando el método de resolución. }\end{array}$} & 3.1 & $\mathrm{PEC}_{2}$ & & \\
\hline & 3.2 & & $\mathrm{PEC}_{4}$ & \\
\hline \multirow{2}{*}{$\begin{array}{l}\text { 4. } \begin{array}{l}\text { Comprender la semántica de la lógica de } \\
\text { enunciados y de predicados y saber aplicarla en } \\
\text { la validación de razonamientos. }\end{array} \\
\end{array}$} & 4.1 & PEC 2 & & \\
\hline & 4.2 & & $\mathrm{PEC}_{4}$ & \\
\hline $\begin{array}{l}\text { 5. Adquirir los conocimientos básicos de la lógica } \\
\text { booleana y aplicarla en circuitos lógicos. }\end{array}$ & 5.1 y 5.2 & $\mathrm{PEC}_{2}$ & & \\
\hline $\begin{array}{l}\text { 6. Conocer el lenguaje de teoría de conjuntos básica } \\
\text { y saber relacionarla con la lógica. }\end{array}$ & 6.1 y 6.2 & $\mathrm{PEC}_{4}$ & & \\
\hline
\end{tabular}

Tabla 1. Tabla de interrelación de Lógica

\section{LAS ACTIVIDADES Y EL PROCESO DE EVALUACIÓN}

Uno de los procesos educativos más complejos es la evaluación de las competencias. A menudo, el concepto de competencia es un término difícil de mostrar, medir y cuantificar, para el docente y también de compleja comprensión para el estudiante. Una competencia difiere de un objetivo puesto que ésta supone describir el nivel o grado de suficiencia que una persona es capaz de desarrollar para poner en práctica una actividad valorando una combinación de atributos, conocimientos, aptitudes y destrezas. Esta complejidad puede ser mayor en el caso de centros que ofrecen enseñanza a distancia puesto que es necesario explicitar claramente cómo se trabajan habilidades y destrezas a través de entornos de aprendizaje virtuales donde la comunicación se establece de forma asíncrona y sin coincidencia presencial con el profesorado. Para el estudiante a distancia la explicitación y concreción del proceso de adquisición de competencias y su evaluación les permite comprender el resultado de aprendizaje final.

Para reducir la dificultad que supone el proceso de evaluación basado en competencias es importante establecer unos criterios de evaluación claros y concisos que tengan su correspondencia en cada una de las competencias indicadas en la asignatura. Esto implica, para cada actividad, definir las competencias que se pretende que el estudiante adquiera y cómo se evalúan. Es decir, es necesario indicar 
los criterios e indicadores en base a los que se evalúan las competencias adquiridas por cada estudiante.

En el caso de Lógica, para cada una de las actividades se ha trabajado de forma concisa especificando los criterios generales de evaluación y los indicadores en que se concretan. También se indica la/s competencia/s a trabajar y, obviamente el enunciado. A modo de síntesis, se indica este proceso en una de las primeras PECs (tabla 2).

\begin{tabular}{|c|c|c|c|}
\hline Competencias & PEC/Actividad & Criterios & Indicadores \\
\hline $\begin{array}{l}\text { Adquirir la habilidad } \\
\text { de formalizar } \\
\text { expresiones del } \\
\text { lenguaje natural } \\
\text { usando lógica de } \\
\text { enunciados. } \\
\text { Capacidad de } \\
\text { construir una }\end{array}$ & $\begin{array}{l}\text { PEC } 1 . \\
\text { Actividad } 1\end{array}$ & $\begin{array}{l}\text { La correcta } \\
\text { formalización de } \\
\text { cada frase con } \\
\text { lenguaje de lógica } \\
\text { de enunciados, } \\
\text { usando los átomos } \\
\text { dados. }\end{array}$ & $\begin{array}{ll}\text { - } & \begin{array}{l}\text { Identificación de la } \\
\text { conectiva principal. }\end{array} \\
\text { - } & \begin{array}{l}\text { Identificación de las } \\
\text { otras conectivas. }\end{array} \\
\text { - } & \begin{array}{l}\text { Aplicación } \\
\text { correcta de las } \\
\text { conectivas lógicas } \\
\text { correspondientes. }\end{array}\end{array}$ \\
\hline $\begin{array}{l}\text { deducción natural } \\
\text { para validar un } \\
\text { razonamiento en } \\
\text { lógica de enunciados. }\end{array}$ & $\begin{array}{l}\text { PEC } 1 . \\
\text { Actividad } 2 \\
\text { Actividad } 3\end{array}$ & $\begin{array}{l}\text { A partir de las } \\
\text { premisas y la } \\
\text { conclusión de un } \\
\text { razonamiento } \\
\text { en lógica de } \\
\text { enunciados } \\
\text { es necesario } \\
\text { construir } \\
\text { satisfactoriamente } \\
\text { una demostración } \\
\text { con el método de } \\
\text { deducción natural } \\
\text { y escribirla en } \\
\text { el Asistente } \\
\text { siguiendo las } \\
\text { explicaciones del } \\
\text { módulo 1. }\end{array}$ & 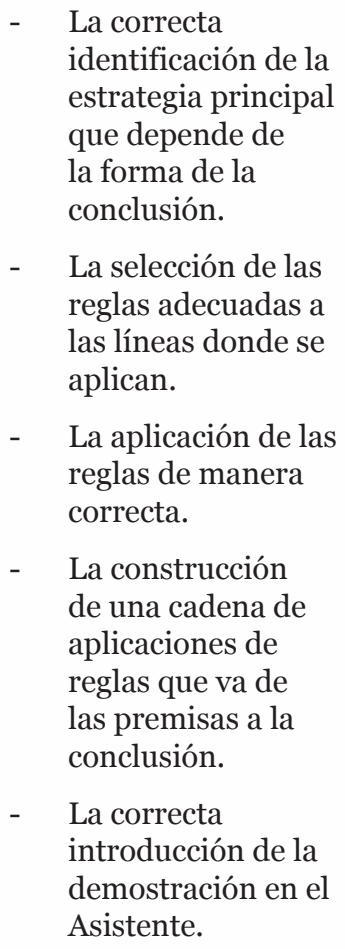 \\
\hline
\end{tabular}

Tabla 2. Relación de competencias, criterios e indicadores para la PEC 
Este proceso se realiza de forma iterativa con todas y cada una de las actividades de evaluación continua que forman parte de la asignatura. Cada actividad especifica en su enunciado las competencias que se trabajan, los criterios que aplican el profesorado en su evaluación y también los indicadores que muestran la concreción de los criterios de evaluación. Como se puede observar en la tabla 2, los estudiantes disponen de los criterios de evaluación en la propia actividad y, a su vez, el profesorado ya tiene elaborados los indicadores de evaluación para validar cada actividad realizada. Para poder concluir que un estudiante ha adquirido una competencia, deberá superar cada una de las actividades propuestas. En función del trabajo realizado, la calificación obtenida y el nivel que se ha establecido de cada competencia el profesorado puede indicar de forma clara las competencias que cada estudiante va adquiriendo a lo largo del curso o bien, qué competencias debe mejorar para lograr su adquisición. La figura 1 indica en qué actividad/es se trabaja cada competencia y su adquisición.
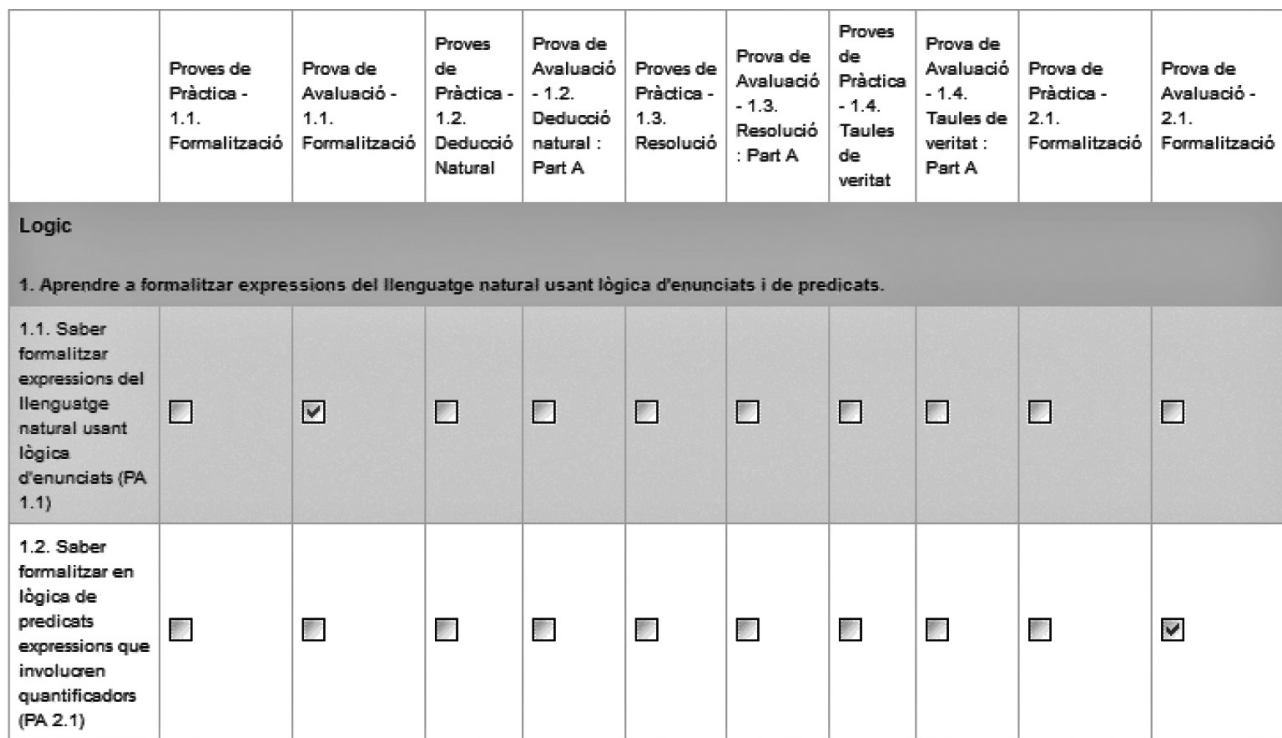

2. Adquirir habilitats de validació de raonaments en lògica d’enunciats i de predicats usant el mètode de deducció natural.

2.1. Copacitat

de construir

ung

demostració de

deducció

natural per

validar un

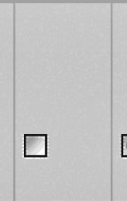

田

Figura 1. Relación de competencias y actividades 
En base a las competencias, criterios e indicadores establecidos el profesorado proporciona a cada estudiante un feedback personalizado de la actividad realizada en base a las competencias establecidas en cada actividad. El feedback que se proporciona y cómo se lo hace puede ser la clave para una mayor comprensión para el estudiante del concepto de competencia en su proceso de aprendizaje.

\section{EL FEEDBACK Y EL RESULTADO DE APRENDIZAJE}

Como se ha comentado en secciones previas, el feedback es una de las claves en cualquier proceso de evaluación. Éste proporciona al estudiante una información más detallada y personalizada sobre su proceso de aprendizaje. En el caso de Lógica, por ejemplo, ayuda al estudiante a conocer el fallo del procedimiento en un ejercicio de matemáticas, en un proceso de formalización del lenguaje, en la validación de un predicado, etc.

El uso de la herramienta "Asistente" provee al estudiante de un feedback inmediato en cada paso del desarrollo de una actividad. Este feedback consiste en la comunicación al estudiante de si el paso es correcto. En el caso de no ser correcto se le proporcionan un conjunto de orientaciones (Huertas et al., 2011). En consecuencia puede mejorar y comprender el procedimiento mediante el cual se resuelve un ejercicio o actividad. Para solventar el error suele consultar los materiales didácticos para revisar de nuevo la aplicación de la teoría en la práctica.

Mediante el feedback y la evaluación formativa el estudiante va adquiriendo un conjunto de habilidades y destrezas que forman parte de una o varias competencias concretas. El resultado final del proceso de enseñanza y aprendizaje, por tanto, se indica al estudiante siguiendo el conjunto de competencias definidas en el plan docente de la asignatura que además han sido concretadas en sus actividades. Al finalizar la asignatura el profesorado de Lógica, partiendo de los contenidos explicitados anteriormente (tabla 1 y figura 1) y del resultado global de las actividades realizadas, indica a cada estudiante el conjunto de competencias que han ido adquiriendo al finalizar el curso (figura 2). 


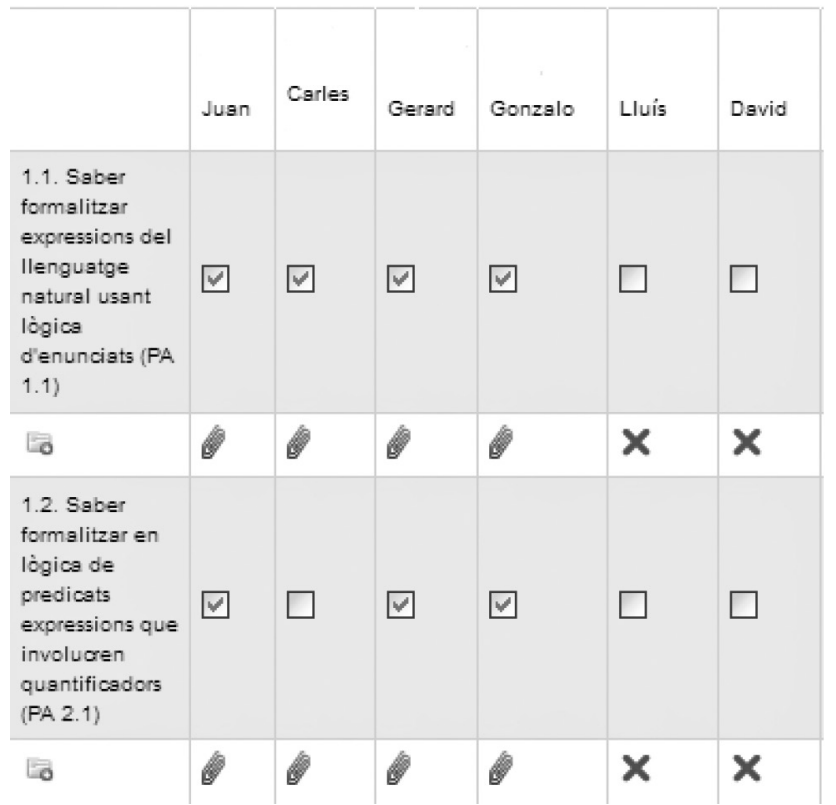

Figura 2. Indicación de competencias a cada estudiante

El profesor no sólo indica la calificación obtenida sino que además proporciona al estudiante un breve resumen de su proceso de aprendizaje basado en las competencias establecidas al inicio de la asignatura. A modo de ejemplo, se indica a cada estudiante si la competencia Adquirir la habilidad de formalizar expresiones del lenguaje natural usando lógica de enunciados ha sido finalmente adquirida mediante las cuatro actividades trabajadas habiendo demostrado su capacidad de formalizar expresiones. Así, para cada una de las competencias establecidas, se le indica al estudiante la misma información dependiendo de las competencias adquiridas mediante su proceso de aprendizaje. Además, también se incluyen referencias a las competencias del grado. De esta manera el profesorado considera que el estudiante puede entender y valorar mejor su proceso de aprendizaje basado en competencias con respecto a la propia asignatura y la totalidad del grado que cursa.

\section{VALORACION GLOBAL}

Para analizar el proceso de interrelación que se ha realizadoy conocer la valoración de los estudiantes de Lógica con respecto a la explicitación de competencias y su evaluación se diseñó un cuestionario que fue enviado por correo electrónico a 50 
estudiantes de la asignatura. Para obtener también una valoración por parte del profesorado implicado se realizaron varias entrevistas.

El cuestionario elaborado para los estudiantes estaba formado por 20 preguntas divididas en diferentes bloques que hacen referencia a las actividades propuestas, al proceso de evaluación formativa, a las calificaciones obtenidas y al feedback proporcionado en base a las competencias establecidas. El cuestionario fue anónimo, de respuesta voluntaria, con preguntas de respuesta abierta y otras siguiendo la escala Likert y fue contestado por 29 estudiantes.

\section{Valoración de los estudiantes}

Los resultados obtenidos del cuestionario indican que los estudiantes consideran que las actividades de evaluación continua están bien planteadas y su enunciado es coherente con respecto a las competencias que se trabajan y cómo se evalúan siguiendo los criterios de evaluación establecidos en cada actividad.

El 86\% de los estudiantes indican que las PECs les ayudan en su proceso de aprendizaje basado tanto en la adquisición de competencias como de conocimientos. El 93\% de los estudiantes indican que han realizado ejercicios de autoevaluación para posteriormente mejorar la PEC. Este dato nos muestra que el proceso de evaluación formativa ayuda al estudiante a evaluar sus destrezas y habilidades respecto a la asignatura. Por consiguiente, dicho proceso ayuda al estudiante a ver los errores de procedimiento y mejorarlos en cada actividad. En este sentido, un $80 \%$ indica que la realización de las PECs les da la oportunidad de evaluar sus fortalezas y debilidades en Lógica y les permite mejorar algunos errores de procedimiento que han realizado durante el curso, consultando las unidades didácticas pertinentes. A pesar de esta buena valoración, los estudiantes indican que seguir una evaluación formativa les conlleva más trabajo y dedicación a la asignatura, puesto que han tenido que reelaborar algunas actividades. Este resultado nos indica que será necesario revisar y modificar algunas actividades partiendo del trabajo que supone para el estudiante seguir una evaluación formativa.

El 86\% de los estudiantes considera que el sistema de graduación y evaluación para cada competencia es adecuado y les permite visualizar semanalmente su progresión en la asignatura. Así mismo un $67 \%$ de los estudiantes indica que la realización de las actividades es muy útil para preparar el examen final.

En lo referente al feedback proporcionado en cada actividad, el $80 \%$ de los estudiantes lo valora muy positivamente indicando, además, que les ayuda a avanzar 
en la adquisición de competencias y a comprender mejor qué supone adquirir una competencia. En consecuencia, se concluye que la inclusión de las competencias en cada actividad junto con sus criterios de evaluación, siguiendo un modelo de evaluación formativa basado en el feedback personalizado, ha sido bien valorado por los estudiantes y les ayuda a entender el proceso de adquisición de competencias.

\section{Valoración del profesorado}

Del análisis de las entrevistas se desprende una valoración positiva por parte del profesorado del área. Éste indica que el proceso de interrelación entre las competencias de grado, de asignatura, las actividades y el proceso de evaluación es un proceso muy laborioso que debe realizarse minuciosamente. Las competencias que se trabajan en la asignatura están definidas en el plan docente, pero destacan que el proceso de relación de dichas competencias con cada actividad y su evaluación ha supuesto un análisis continuo para ajustar y clarificar el proceso de trabajo de cada competencia. El profesorado constata la importancia de un modelo de evaluación formativa para favorecer e incentivar la adquisición de habilidades. Consideran que la evaluación formativa permite al estudiante revisar y repasar los conceptos y prácticas de forma continua para entender y consolidar algunas habilidades complejas que se solicitan en la asignatura. Este proceso les ha supuesto añadir algunos recursos de autoevaluación en la asignatura, replantear las actividades y también relacionar de forma directa las unidades didácticas con ellos. Aún así destacan que es un valor añadido para el estudiante puesto que les permite identificar sus puntos débiles y mejorarlos.

En referencia al feedback y al resultado de aprendizaje el profesorado indica que el hecho de establecer a priori los indicadores de evaluación de cada actividad les facilita en gran parte el proceso de calificación y emisión de feedback cualitativo. Por un lado, les permite proporcionar un feedback centrado en los indicadores definidos facilitando de forma concisa el resultado de la actividad realizada. Por otro lado, les facilita y ayuda a indicar a cada estudiante las competencias que se van trabajando y, por consiguiente, que se van adquiriendo de forma progresiva.

Aunque la valoración global del profesorado es positiva, constatan la gran dedicación inicial de trabajo que supone realizar este proceso de revisión. Aún así, consideran que después de varios cursos de implementación de los grados siguiendo el EEES, es necesario realizar una revisión y análisis de las competencias establecidas, cómo se trabajan, cómo se evalúan y cómo se trasladan al estudiante en sus asignaturas para mejorar y consolidar el proceso de enseñanza y aprendizaje basado en competencias. 


\section{CONCLUSIONES}

En este artículo se ha presentado el proceso realizado con el objetivo de alinear las competencias de grado con las de asignatura, sus actividades, criterios de evaluación y resultados de aprendizaje. Este proceso se ha realizado en la asignatura de Lógica y se propone extenderlo a otras asignaturas para analizar y revisar el proceso de enseñanza y aprendizaje basado en competencias y hacerlo más clarificador. El proceso de interrelación realizado permite tanto a estudiantes como a docentes mostrar el proceso de adquisición de competencias en el que se basa el proceso de Bolonia. Si bien la implementación de los grados ha supuesto un primer paso hacia la formación de profesionales competentes, se hace necesario revisar y analizar dicho proceso a escala más reducida e institucional para valorar cómo se está realizando el proceso de enseñanza y aprendizaje basado en competencias en cada universidad.

La principal aportación de este artículo es el proceso de revisión e interrelación que se ha llevado a cabo en el caso de Lógica puesto que es aplicable a otras asignaturas y, sobre todo, para docentes interesados en clarificar que implica la adquisición de competencias. No obstante, este proceso puede no ser extrapolable directamente a otras asignaturas dada la especificidad y complejidad de algunas áreas de conocimiento o tipología de contenidos. En dichos casos será necesario analizar cada caso para establecer el proceso de alineación más adecuado. Aún así a través del proceso comentado en este artículo, otros docentes pueden revisar sus asignaturas a modo de análisis y reflexión tras los primeros años de implantación del grado. La experiencia realizada en el caso de Lógica ha sido bien valorada tanto por los docentes como los estudiantes implicados en el proceso. Aunque los docentes indican que trabajar competencias representa un trabajo mayor y más laborioso, coinciden en que la inmersión inicial supone una ventaja posterior durante el curso para evaluar a sus estudiantes. Por otra parte, la valoración de los estudiantes mostrada a través del cuestionario realizado indica una mayor comprensión de lo que supone adquirir unas competencias concretas y valoran de positivamente el detalle reflejado tanto en las actividades como en su proceso de evaluación.

Actualmente y siguiendo el trabajo realizado, se propone extender este proceso de análisis y revisión a otras asignaturas del mismo grado para realizar un análisis global de las competencias que se estipulan en las memorias de grado y su concreción en cada asignatura. También sería conveniente analizar de forma más concreta los itinerarios que se ofrecen en cada titulación en relación a las competencias específicas de cada uno de ellos. Un profundo análisis de revisión de la implantación de los grados puede llegar a mejorar el proceso de enseñanza y aprendizaje basado 
en competencias situando tanto al estudiante como a las actividades que realiza en el centro de un modelo educativo basado en competencias.

\section{NOTAS}

1. Universitat Oberta de Catalunya. http://www.uoc.edu/

2. Declaración de Bolonia (1999) [web]. Disponible en: http://www.crue.org/export/ sites/Crue/procbolonia/documentos/antecedentes/2. Declaracixn de Bolonia.pdf [consulta 2013, 1 de abril].

\section{REFERENCIAS BIBLIOGRÁFICAS}

Avargil, S.; Herscovitz, O.; Dori, Y. (2012). Teaching thinking skills in contextbased learning: Teachers challenges and assessment knowledge. Journal of Science Education and Technology 21, (207-225).

Burgos, D. (2006). The structure and behavior of virtual communities engaged in informal learning about elearning standards. Madrid: Universidad Europea de Madrid.

Chen, H.; Wigand, R.; Nilan, M. (1999). Optimal experience of web activities. Computers in Human Behavior, 15 (5), (585608).

Díaz, F. (2002). Didáctica y currículo: un enfoque constructivista. Ediciones de la Universidad de Castella-La Mancha.

Fainholc, B. (2008). De cómo las TICs podrían colaborar en la innovación sociotecnológico-educativa en la formación superior y universitaria presencial. RIED. Revista Iberoamericana de Educación a Distancia, 11 (1), (53-79).

González, J.; Wagenaar, R. (2003). Tuning educational structures in Europe. [en línea] Disponible en: http://www.unideusto.org/tuningeu/images/stories/ Publications/Tuning phase1 full document.pdf. [consulta 2013, 24 de marzo].

Goodrich, H. (2000). Using Rubrics To Promote Thinking and Learning. Journal of Educational Leadership, 57 (5), (13-18).
Halttunen, K.; Sormunen, E. (2000). Learning information retrieval through an educational game: Is gaming sufficient for learning? Education for Information, 18, (289-311).

Huertas, A.; Humet, J. M.; López, L.; Mor, E. (2011). The SELL Project: a Learning Tool for E-learning Mathematical logic. In Blackburn, P. et al. (ed.). Third International Congress on Tools for Teaching Mathematical logic (TICTTL 2011), LNAI 6680, 123-130. Springer, Heidelberg

Iram, S.; Al-Jumeily, D.; Lunn, J. (2011). An Integrated Web-Based e-Assessment Tool. Developments in E-systems Engineering (DeSE), (271-275). doi:10.1109/ DeSE.2011.111

JISC. (2007). Effective Practice with e-Assessment: An overview of technologies, policies and practice in further and higher education. [en línea] Disponible en: http://www.jisc.ac.uk/media/documents/themes/elearning/effpraceassess.pdf [consulta 2013, 24 de marzo].

Lytras, M.; Sicília, M. A. (2005). On the representation of change according to different ontologies of learning. International Journal of Learning and Change, 1 (1), 66-79.

López-Pastor, V. M. (2010). El papel de la evaluación formativa en la evaluación por competencias: aportaciones de la Red de Evaluación formativa y Compar- 
tida en Docencia Universitaria. REDU. Revista de Docencia Universitaria, 9 (1), (159-175).

Marín, V.; Cabero, J.; Barroso, J. (2012). La rúbrica de evaluación en el proceso de formación del docente universitario. La propuesta del proyecto DIPRO 2.0. Revista Educar, 48 (2), 347-364.

Moskal, B. M. (2000). Scoring rubrics: what, when and how? Practical Assessment, Research \& Evaluation, 7 (3). [en línea] Disponible en: http://PAREonline.net/ getvn.asp? $\mathrm{v}=7 \& \mathrm{n}=3$. [consulta 2013, 24 de marzo].

Mostrom, A.; Blumberg, P. (2012). Does learning-centred teaching promote grade improvement?. Journal of Innovative Higher Education, 37, (1-9).

Navarro, J.; Ortells, M.; Martí, P. (2011). Las "rúbricas de evaluación" como instrumento de mejora educativa. XI Jornada sobre Aprendizaje Cooperativo y IV Jornada sobre Innovación en la Docencia. [en línea] Disponible en: http://spieu. uji.es/JACMain/11/EVAL/6.pdf [consulta 2013, 24 de marzo].

Nicol, D. (2006). Increasing success in first year courses: Assessment re-design, self-regulation and learning technologies. 23rd Annual ASCILITE Conference: Who's learning? Whose technology? Sydney.

Richey, R.; Fields, D.; Foxon, M. (2011). Instructional design competencies: The standards. Clearninghouse on Information and Society.

Scriven, M. (1967). The methodology of evaluation. In Tyler, R. W.; Gagne, R. M.; Scriven, M. (eds.). Perspectives of $\mathrm{cu}^{-}$ rriculum evaluation, (39-83). Chicago: Rand-McNally.

Scriven, M. (1974). Prose and cons about goal-free evaluation. Evaluation Comment, 3, (1-4).

Sharpe, R.; Benfield, G.; Roberts, G. (2006). The undergraduate experience of blended e-learning: a review of UK literature and practice. A Report to the Higher Education Academy. [en línea] Disponible en: http://www.heacademy.ac.uk/ assets/documents/teachingandresearch/sharpe benfield roberts francis.pdf [consulta 2013, 24 de marzo].

Tierney, R.; Simon, M. (2004). What's still wrong with rubrics: focusing on the consistency of performance criteria across scale levels. Practical Assessment, Research \& Evaluation, 9, (2). [en línea] Disponible en http://PAREonline.net/ getvn.asp? $v=9 \& n=2$ [consulta 2013, 24 de marzo].

Torres, J.; Perera, V. (2010). La rúbrica como instrumento pedagógico para la tutorización y evaluación de los aprendizajes en el foro online en educación superior. $P i$ xel-Bit. Revista de Medios y Educación, 36, (141-149).

\section{PERFIL ACADÉMICO Y PROFESIONAL DE LOS AUTORES}

Ana Elena Guerrero Roldán. Licenciada en Pedagogía por la Universidad Ramon Llull. Doctora en la Sociedad de la Información y el Conocimiento por la UOC. Profesora en el área de Capacitación digital de los estudios de Informática, Multimedia y Telecomunicación de la UOC. Coordina asignaturas transversales sobre competencias TIC en diferentes grados. Participa en diferentes proyectos de investigación sobre la influencia de las TIC en la educación a distancia, el proceso de aprendizaje y evaluación en entornos virtuales.

E-mail: aguerreror@uoc.edu 
M. Antonia Huertas. Doctora en Ciencias Matemáticas por la Universidad de Barcelona. Profesora en el área de Fundamentos Matemáticos de los estudios de Informática, Multimedia y Telecomunicación de la UOC. Profesora de los programas de Doctorado de la Sociedad de la Información y el Conocimiento y de e-Learning de la UOC. Sus intereses de investigación incluyen lógica, representación del conocimiento, aprendizaje mejorado por la tecnología y e-learning de las matemáticas.

E-mail: mhuertass@uoc.edu

Enric Mor. Ingeniero en Informática por la Universitat Politècnica de Catalunya (UPC). Doctor en Sociedad de la Información y el Conocimiento por la Universitat Oberta de Catalunya (UOC). Desde 1998 es profesor de los Estudios de Informática, Multimedia y Telecomunicación de la UOC. Su docencia incluye diferentes cursos de grado y postgrado en el ámbito de la interacción persona-ordenador y accesibilidad. Sus principales áreas de investigación son la interacción persona-ordenador, la accesibilidad y el aprendizaje potenciado por la tecnología.

E-mail: emor@uoc.edu

M. Elena Rodríguez. Licenciada en Informática por la Universitat Politècnica de Catalunya. Doctora en Ciencias de la Computación por la Universidad de Alcalá. Profesora de los Estudios de Informática, Multimedia y Telecomunicación de la UOC donde coordina diferentes asignaturas relacionadas con bases de datos. Participa en diferentes proyectos de investigación sobre el desarrollo de modelos de representación del conocimiento como medio para mejorar el aprendizaje a través de la tecnología.

E-mail: mrodriquezgo@uoc.edu

DIRECCIÓN DE LOS AUTORES

Universitat Oberta de Catalunya (UOC)

Estudios de Informática, Multimedia y

Telecomunicación

Rambla del Poblenou, 156

o8010 Barcelona, España

Fechas de recepción del artículo: 11/01/13

Fecha de aceptación del artículo: 29/04/13 


\section{Como citar este artículo:}

Ana-Elena Guerrero-Roldán, A. E.; Huertas, M. A.; Mor, E.; Rodríguez, M. E. (2013). Explicitando la interrelación entre las actividades de aprendizaje, el proceso de evaluación y la adquisición de competencias. RIED. Revista Iberoamericana de Educación a Distancia, volumen 16, nº 2, pp. 127-146. 